\title{
Benchmarking a computerized test of immediate verbal memory
}

\author{
Rochelle E. Tractenberg and Colin E. Freas \\ Georgetown University, Washington, D.C.
}

\begin{abstract}
We modified the traditional (verbal) digit span task for administration via computer and the Internet. This online version collects data on the floor and ceiling of a subject's span capacity, rather than generating a rough estimate of capacity based on a 50\% success rate, as the traditional version does. We compared the two versions within adult subjects in two cohorts: college-age normal readers and college-age reading-disabled readers. To explore the reliability of the online version as a research tool, we employed the Bland-Altman approach to examine agreement between instruments. The online version yielded spans similar to those yielded by the traditional version, tending toward smaller values at the high end and larger values at the low end of span sizes, in the typical readers. It differentiated between the better and poorer readers reliably, and to the same extent as does the verbal version. The online version of the digit span task is comparable to the traditional version in assessing verbal span capacity; the code with which to implement the task is available at www.psychonomic.org/archive.
\end{abstract}

One of the most consistently used tests of immediate verbal memory is the digit span task from the Wechsler IQ batteries (WAIS-R and WISC-R; Wechsler, 1981, 1983). In this task, the experimenter reads lists of single digits aloud to subjects, who are asked to repeat the same list either verbatim (forward) or in the opposite order to the one in which they were given (reverse). The list of digits starts with two or three, and grows by one when the subject responds correctly on at least one of two lists at each length, and in both recall directions (forward and reverse). If the subject makes an error, in order or content, on both trials, the list length preceding this one is taken as the subject's digit span, indicative of his or her verbal memory capacity.

There are three distinct disadvantages to the traditional, verbal ("out loud") format of this test. First, a verbal task is biased against individuals with hearing or receptivelanguage deficits; this is important in the study of reading disability as well as in studies of elderly persons who may have reduced hearing capabilities. Another disadvantage is that individual differences in subjects might be confounded, or compounded, by individual differences in experimenters. The traditional digit span task involves an experimenter reading a list of digits out loud at a rate of one per sec. Without a timer or metronome, replicating this rate for every digit and every subject could very well be impossible, and it has been noted that presentation rate may affect performance (see Gathercole \& Baddeley, 1993; Penney, 1975). Finally, within the traditional task protocol, very few data points are collected per person (i.e., only two trials are possible at the maximum span size). In other words, traditional digit span is a single es- timate of a subject's immediate verbal memory capacity: the largest string size at which at least one of two trials was successful.

Trojano, Chiacchio, De Luca, Fragassi, and Grossi (1994) compared patients with diagnoses of probable Alzheimer's-type dementia with control (nondemented) individuals on the visuospatial (i.e., nonverbal) correlate of the digit span, the Corsi Block test (Milner, 1974). They used one of two criteria for success at any set sizesuccess on one of two trials (the traditional digit span task criterion, "lenient") or on three of five trials ("hard"). They found that spans obtained with the lenient criterion were higher for both groups than were spans based on the harder criterion, and proposed that the harder criterion could increase the sensitivity of the task (p. 862). Although their data come from a visuospatial memory task, the conclusions of the 1994 study exemplify a well-known fact in assessment: More precise estimates arise from a more extensive test of the system in question.

The purpose of this article is to describe the design and benchmarking of an online alternative to the traditional test of verbal immediate memory. We specifically developed the test so that it would (1) be accessible for both hearing and hearing-impaired subjects, (2) have timing controlled by a computer, and (3) collect data on the floor and ceiling of a subject's memory capacity via an updown staircase algorithm.

The up-down method has historically been used in psychoacoustic (Levitt, 1971) and vision (Tyler \& Gorea, 1986) studies, and is used generally throughout psychophysics. Levitt describes up-down testing as a sequential task in which the procession of the experiment is de-

R. E. Tractenberg, ret7@georgetown.edu 
pendent on responses made during the experiment - that is, the level of the stimulus (easier or harder to respond to) to which a subject is exposed depends on the subject's responses. As noted, the two-trial methodology used in the traditional digit span task derives an estimate of span size as the level preceding that at which failure on both trials is observed once. In contrast, the up-down methodology derives span size from an average based on levels at which a subject will consistently succeed and fail.

A computerized version of the digit span task with the up-down approach obviates the disadvantages of the traditional version described above. It can provide visual or (with a sound card and trivial modification) auditory stimuli, presented at a constant rate controlled by the computer. The program can generate, and keep track of, random numbers in set sizes as specified, so that the result of any trial (i.e., success or failure) can immediately result in a new trial with random numbers in the correct set sizes. The program can record both the target strings and the response strings, the comparison of which would allow exploration of primacy or recency effects (if desired).

This article presents data collected from both traditional and online versions of the digit span task (forward and reverse conditions) in two cohorts. The two groups were (1) normally reading four-year university students and (2) reading-disabled students identified by the community college learning disabilities staff - groups known to differ in their verbal memory capacity. It is a well-documented finding in research involving verbal immediate memory that reading-disabled individuals perform worse on a digit span task than their normally reading peers (see Torgesen, 1996, for a review). The data were collected to address two issues in the description of this new online digit span task: whether the online version agrees with the verbal version with regard to estimated span size and whether the expected difference in verbal memory performance of disabled readers relative to normal readers can be observed with both versions of the task.

\section{METHOD}

\section{Subjects}

Two cohorts of individuals were assessed in a study focused on reading (reported in Tractenberg, 1997) that hypothesized a key role for immediate memory in reading; therefore, the best estimate of immediate memory was sought.

College, typical readers. In this cohort, 27 students (average age, 20.1 years; range, 18-23 years) who were native English speakers (or who had learned English before age 5) were recruited from the subject pool in the Department of Psychology at the University of California, Irvine. These subjects (12 men, 15 women) received course credit for their participation. As indicated above, the memory tasks described below were administered as part of a larger battery, so subjects' participation lasted up to $2 \mathrm{~h}$. However, the two memory tasks described here took a total of approximately $25 \mathrm{~min}$.

College, disabled readers. This cohort included 10 readingdisabled adults (average age 31.2 years; range 19-47 years) who were native English speakers (or who had learned English before age 5) and were recruited from three local community colleges. All subjects met the California Community College criteria for a learning disability (see Mellard, 1990), and their eligibility for this study was based on the following criteria. Subjects were to have (1) normal $(>90)$ full scale IQ (WAIS-R); verbal IQ lower than performance IQ by at least 5 points; (2) a discrepancy (of at least three grade levels) between reading and other achievement test scores (WRAT-R, WRAT, or Woodcock-Johnson); ${ }^{1}$ (3) reading as the primary target of the specific learning plan established for them by the schools' learning disabilities specialists; and (4) a referral as "reading disabled" by the schools' learning disabilities specialists.

Individuals in this cohort had no history of traumatic brain injury or general learning disability, hearing impairment, or lack of adequate instruction opportunity (1 subject had not graduated from high school, but had been attending community college classes over a period of 10 years). Their average age was 31.2 years (range, 19-47 years), and the average reading level, based on grade-level equivalents for test scores as given in academic files, was just over 5 th grade (5.25). These subjects ( 4 men, 6 women) received $\$ 15$ for their participation. Performance IQ scores were collected from the existing academic files of the subjects as part of the procedure of confirming each individual's status as having "normal" IQ and evidence of a reading disability. Local institutional review boards approved the data collection.

\section{Materials}

The instructions for the two versions of the digit span task appeared on a single screen for the online version, and were printed on a single sheet of paper for the verbal version. These were given to the subjects to read and were also read aloud by the experimenter. Both sets of instructions are presented in the Appendix.

Online digit span. A program was originally written (by R.E.T.) for the Macintosh computer in Think C (Symantec, 1993) and later adapted (by C.E.F.) to be platform independent and Internet accessible (www.psychonomic.org/archive). The program first presented written instructions for the task, which disappeared when a subject clicked the mouse on a button labeled DONE. When the button was clicked, the task began. Prior to each trial, the subject saw the messages "Ready" and then "Go," for 500 msec each. The program randomly selected single digits between 0 and 9 and presented them on the screen for $500 \mathrm{msec}$. A digit disappeared and another replaced it, and so on, until the end of that string was reached. The first string was always three digits long. After the final digit in a string, a recall cue (either "Forward" or "Backward") appeared for $500 \mathrm{msec}$. A dia$\log$ box then appeared, prompting the subject to "Enter sequence of numbers," and hit $<$ Return $>$ (or the button labeled ENTER, using the mouse). Subjects typed their (entire) responses, using either the top row of numbers on the keyboard or the keypad, and were instructed to type " $\mathrm{x}$ " for digits they did not recall. Subjects were therefore in control of the initiation of each trial. Once the forward sequences ended, the message "Backward sequences" appeared for $500 \mathrm{msec}$. All forward sequences were given together, followed by all reverse sequences.

With the exception of the first set of stimuli, which was always three digits long, the length of each sequence was determined by the subjects' responses. The length of a subsequent string was increased by one digit if the previous response was correct, and was decreased by one digit if the previous response was incorrect (or missing, or " $x$ "). The range of possible string sizes (and spans) was therefore positive, but had no upper limit. If a subject responded correctly, the change in string length was positive, whereas incorrect responses (i.e., at least one element in the response was wrong or in the wrong place) caused the change to be negative. At each such inflection point (going from a correct response to an incorrect one, or vice versa), a counter within the subject's data file was incremented. When the inflection counter reached six, that segment of the task ended and either reverse sequences were given or the final message was displayed ("Thank you"). The online digit span was calculated by averaging the span sizes at each of the six inflection points in each direction (see the Appendix for example responses).

Verbal digit span. The traditional (verbal) task involved strings of digits ranging in size from 2 to 11 . The same stimuli were used for each subject, as in the WAIS-R digit span task (Wechsler, 1981). The materials for this task were therefore two strings at each set size 
to be recalled verbatim, followed by the reversed order (as per the WAIS manual). The cue to recall was the spoken word "frontward" or "backward." For each subject, errors in recall were recorded on sheets with the stimuli lists printed on them. The string size preceding that on which both of two trials were failed was recorded as the span size (for that direction) for each subject.

Statistical method. Association describes a general linear relationship between two variables $(x, y)$, and the strength of this association is estimated in the generation of a correlation coefficient (Stevens, 1996). Altman and Bland (1983) described a method for comparing a pair of methods for measurement in a clinical setting. Their method for assessing agreement, rather than association, of two different ways of measuring the same construct is qualitative, and involves plotting the difference between the two measurements on the $y$-axis against the average of the two measurements on the $x$-axis (Bland \& Altman, 1986). Altman and Bland (1983) suggested that "limits of agreement," reflecting tolerance for deviations in the two measurements, could be derived from the mean and standard deviation of the differences between the two measures. Generally, agreement is best if the mean value of the difference is zero. In terms of estimating memory capacity (span), the verbal version results in whole numbers, whereas the up-down method can result in nonintegers. Therefore, perfect agreement is unlikely between the two methods for assessing span that are described here. We adapted the argument for limits of agreement with a "one-unit" range around the mean difference. Therefore, if the two versions of memory span result in estimates that are less than one unit apart, we would conclude that they agree sufficiently in their assessment of the underlying construct (memory capacity). If the agreement is one unit or more apart, we would conclude that the methods give different estimates of an individual's span.

Because the average of the two measures ( $x$-axis) involves two data points, it is the most precise estimate of capacity that we have for any person in our data set (see, e.g., Nunnally \& Bernstein, 1994, pp. 67, $221)$; since it has two components, each reflecting the construct of interest, it is more precise than either of the two single components could be. Thus, the Bland-Altman plot graphically illustrates the way that two measures differ in their assessment of the same construct (on the $y$-axis) as a function of the best estimate of the construct itself (on the $x$-axis). We hypothesize that the agreement between the two measures should be consistent over all values on the $x$-axis.

\section{Design and Procedure}

Subjects were run individually. The two versions of the digit span task were separated by at least one test of visuospatial immediate memory and a set of totally unrelated tasks (testing reading-specific skills). The traditional version was always administered second.

\section{RESULTS}

Figures 1A and 2A are Bland-Altman plots, so the mean value for the two presentation methods is presented on the $x$-axis. The $y$-axis presents the difference between the online span and the verbal span. It therefore reflects the difference between the two measurements for an individual, and the presentation methods give the same estimate of span (treating span as an ordinal variable) if the $y$-axis value falls inside the interval bounded by (i.e., excluding) -1.0 and 1.0. Reference lines shown on the $y$-axis delineate differences between the two presentation methods that are within 1 point (above or below) - that is, the reference lines show observations where the two methods give the same integer estimate of span (i.e., agree). For interested readers, the association is shown in the simple
A

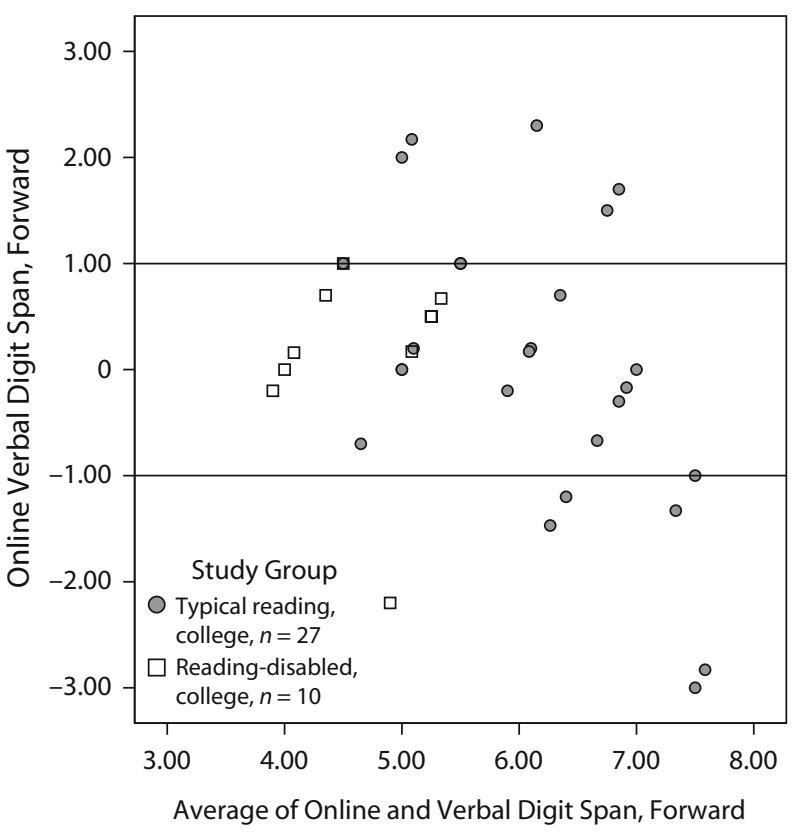

B

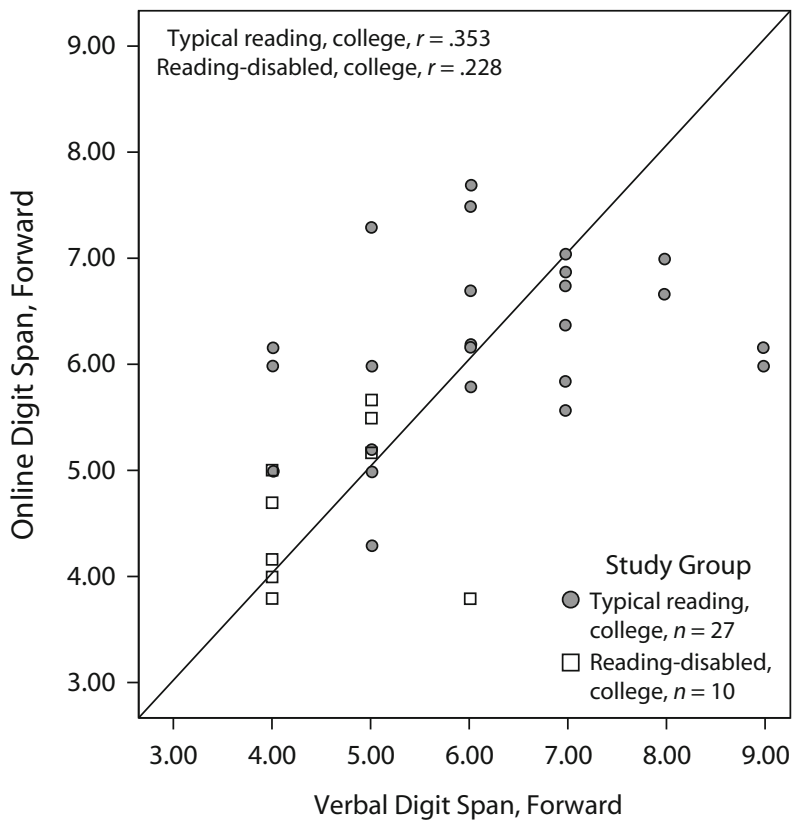

Figure 1. (A) Bland-Altman plot for forward span capacity in two college-age reading cohorts. The Bland-Altman plot shows the average between two measures on the $x$-axis as the best estimate of the "true" value, and the difference between the two measures on the $y$-axis as the estimate of agreement between the two measures. The references lines at \pm 1 represent "agreement" in span estimates, accounting for the fact that the online version does not always yield an integer value. (B) Simple scatterplot of forward span capacity in two college-age reading cohorts. The simple scatterplot shows each of the two measures of forward span on one of the axes. The reference line represents perfect association between span estimates (correlation or slope of a simple linear regression $=1.0$ ). 
scatterplots (Figure 1B for forward, and Figure 2B for reverse), summarized with a Pearson correlation coefficient calculated separately by group.

Figure 1A shows the Bland-Altman plot of the forward span estimates for the two cohorts; Figure 1B shows the simple scatterplot. The online and verbal versions of the forward span gave the same span in $20 / 37$ or $54.1 \%$ of cases. In both cohorts, the proportion of disagreements between the online and verbal forward span estimates (where online span estimates were greater, and where they were less, than the verbal versions) were similar (as can be seen in Figure 1A). What is important from Figure 1A is that although the proportions of observations falling above and below the top/bottom reference lines are similar, observations tend to fall below the lower reference line when average span is higher, whereas observations fall above the top reference line when average span is lower. Figure 1B does not reflect the latter pattern, but does generally reflect a tendency for observations to fall above, more so than below, the reference line representing perfect correlation (slope $=1$ ). The correlation coefficients did not reach significance for either cohort, but more importantly, their squared values reflect a very (surprisingly) small degree of common variance, given that both tests represent verbal memory capacity $(<15 \%)$.

Reverse span estimates using the two methods are shown in the second Bland-Altman plot (Figure 2A) and simple scatterplot (Figure 2B). The two versions of reverse span gave the same value in $23 / 37$ or $62.6 \%$ of cases. One of the cases in which the methods did not agree on the reverse span was with an individual whose verbal span was 10 and online span was 3.7. This observation appears to be an outlier of sorts (i.e., it was not a typo), but the values were so extreme (highest verbal span, among the lowest online spans) that there was no way to conclude whether or not to exclude the observation. Wherever a difference in conclusions hinged on this observation, we have made a note of it.

As with the forward spans, in both cohorts, the proportion of disagreements between the online and verbal forward span estimates (where online span estimates were greater, and where they were less, than the verbal versions) were similar. Figure 2B also reflects a tendency for observations to fall above, more so than below, the reference line representing perfect correlation (slope $=1$; similar to Figure 1B). As with the forward spans, the correlation coefficients did not reach significance for either cohort, and their squared values reflect a very small degree of common variance $(<15 \%$ for typical readers and about $0 \%$ for the disabled readers). However, when the "outlier" in the reverse spans (online, 3.7; verbal, 10) was removed from the typical readers' data, the correlation between the two methods was $.665(p<.001)$, boosting their shared variance in the reverse spans to $44.2 \%$.

Considering the Bland-Altman plots for both cohorts, the likelihood of a disagreement between the verbal and online versions (i.e., a data point falling outside the two
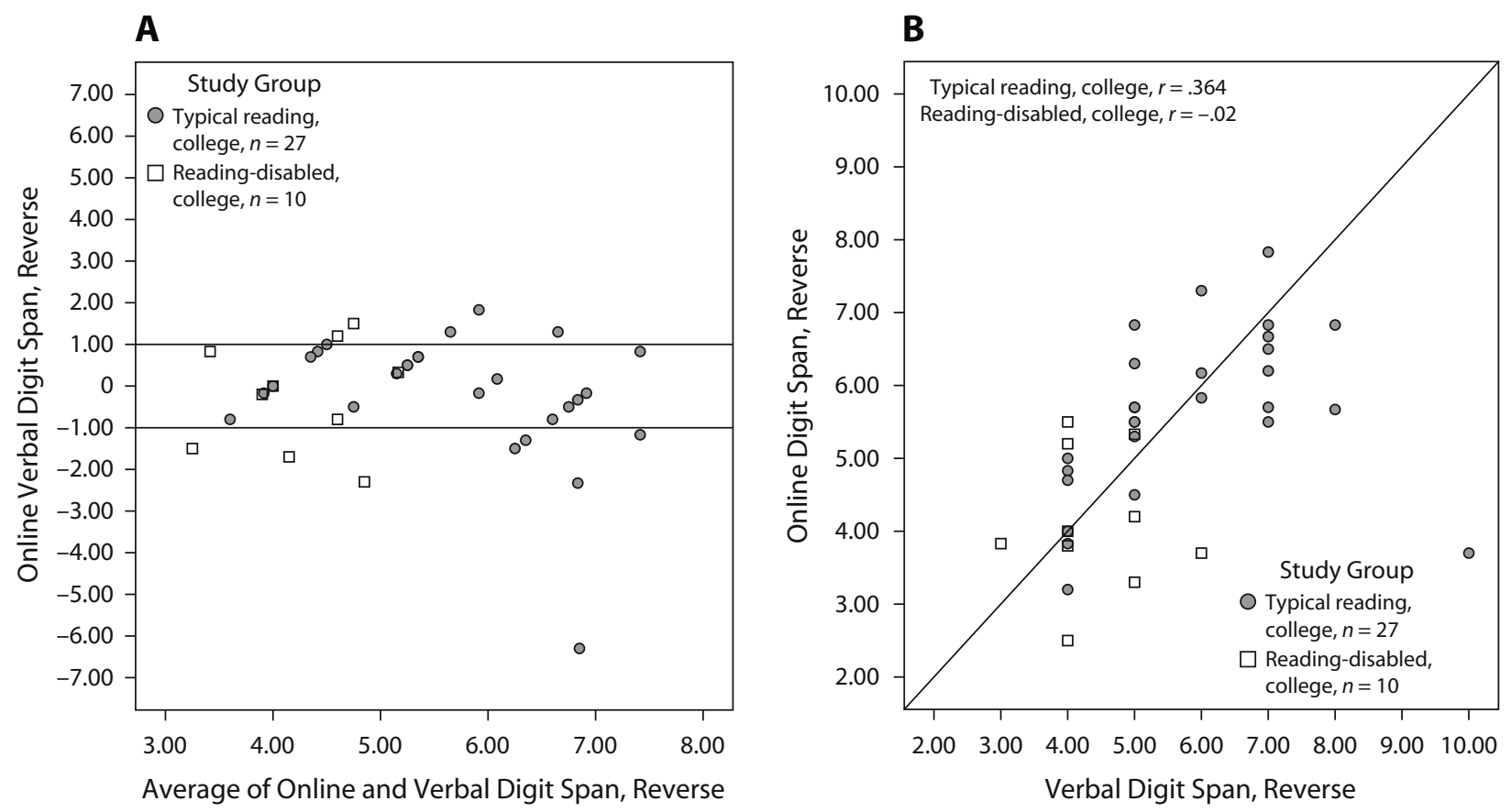

Figure 2. (A) Bland-Altman plot for reverse span capacity in two college-age reading cohorts. The Bland-Altman plot shows the average between the two measures on the $x$-axis as the best estimate of the "true" value, and the difference between the two measures on the $y$-axis as the estimate of agreement between the two measures. The references lines at \pm 1 represent "agreement" in span estimates, accounting for the fact that the online version does not always yield an integer value. (B) Simple scatterplot for reverse span capacity in two college-age reading cohorts. The simple scatterplot shows each of the two measures of reverse span on one of the axes. The reference line represents perfect association between span estimates (correlation or slope of a simple linear regression $=1.0$ ). Without the typical reader "outlier" point, the Pearson correlation coefficient is .689 $(p<.001)$. 
reference lines) was not related to the $x$-axis value for $r e$ verse span [Pearson correlation coefficients: typical readers, $r=-.315$ (without the extreme value, $r=-.256$ ); disabled readers, $r=.124$; both $p \mathrm{~s}>.20]$. Differences in forward span ( $y$-axis values) were not associated in the disabled readers $(r=.058, p>.8)$ and were significantly negatively associated with the average of the verbal and online values ( $x$-axis values) in typical readers $(r=$ $-.523, p<.01)$. This suggests that in typical readers, and for larger forward spans, the online version gives a lower value than the verbal version, whereas for smaller forward spans, the online version gives a higher value. The significance of these correlations is not as important as the shared variance they suggest as estimates- low values for reverse spans and a relatively high value $(27 \%)$ for just the typical readers in the forward span. The scatterplots do not provide information about the relative performances of the two measures of span in each direction-mainly because that is not the purpose or power of a measure of association (i.e., correlation).

Table 1 presents the descriptive statistics for the sample-specific estimates of span by the online and verbal methods, with the performance IQ information about the two cohorts. As noted above, the online method provided a slightly higher average forward span in typical readers than did the verbal version, whereas the reverse span in this method was slightly smaller than that measured by the verbal version. As shown in Figures 1B and 2B, the Pearson correlations calculated between the observed values using these two methods, and separately by group, revealed unexpectedly small associations between spans in both directions [typical readers, forward, $r=.353, p=$ .07 ; reverse, $r=.364, p=.06$ (without outlier, $r=.689$, $p<.001$ ); disabled readers, forward, $r=.228, p=.53$; reverse, $r=-.02, p=.96]$.

We compared the mean spans collected by the two methods across the normal and disabled readers in the two cohorts. The disabled readers were found to have smaller spans by both the online [forward, $t(35)=4.8$, $p<.001$; reverse, $t(35)=3.65, p=.001]$ and verbal [forward, $t(35)=3.14, p<.01$; reverse, $t(29.5)=3.54$, $p=.001]$ methods (the extreme reverse span values did not alter these results, so this observation was included). The groups did not differ significantly on the measures of their Wechsler performance IQs $[t(16)=-0.082$, $p=.21$ ], so the differences between their digit spans cannot be attributed to disparities in general ability, or differential problems with either task method.

Table 1

Verbal Versus Online Digit Spans (Mean $\pm S D$ ) in Typical and Disabled Readers (College Cohorts)

\begin{tabular}{lccc}
\hline & $\begin{array}{c}\text { Typical } \\
\text { Readers } \\
n=27\end{array}$ & $\begin{array}{c}\text { Disabled } \\
\text { Readers } \\
n=10\end{array}$ & $p<$ \\
\hline Verbal forward & $6.07 \pm 1.41$ & $4.60 \pm 0.70$ & .001 \\
Online forward & $6.15 \pm 0.82$ & $4.73 \pm 0.74$ & .001 \\
Verbal reverse & $5.81 \pm 1.55$ & $4.40 \pm 0.84$ & .001 \\
Online reverse & $5.62 \pm 1.14$ & $4.14 \pm 0.96$ & .001 \\
Performance IQ & $37.9 \pm 6.61$ & $40.0 \pm 4.52$ & n.s. \\
\hline
\end{tabular}

\section{DISCUSSION}

On average, the spans for both cohorts in both directions were similar for the online and traditional (verbal) presentations. Furthermore, the expected differences between the memory performance of the normally reading and reading-disabled college-age groups were found using both presentation methods. Importantly, repeatedly testing the maximum span size using the up-down method in the online version seemed to result in a smaller observed span in typical readers with larger spans, whereas the additional tests at the low end of the span size seemed to "boost" lower span sizes in this cohort. This was not generally observed in the disabled readers, however, suggesting that there is a low level of variability in their performance in both directions, whereas typical readers seem to vary more at the extremes (smaller and larger spans). These observations should be followed up with larger sample sizes and possibly with counterbalanced presentations.

The purpose of this study was to describe a new online version of the traditional digit span task that involves more than two trials at an individual's minimal and maximal span sizes; the description was contextualized as a comparison with the results of the traditional two-trial digit span test. The Bland-Altman plots suggest that the results yielded by the traditional and online versions generally agree with regard to forward and reverse spans in independent groups of subjects; the online version also demonstrated the expected discrepancy in the immediate memory capacities of the two college-age groups, a finding that has been reported in many other studies (see Torgesen, 1996, for a review). This discrimination is an important attribute of any memory test; however, we do not believe that a digit span estimate based on the traditional verbal administration should be conceptualized as "true," or that other types of estimates must be equivalent somehow to the estimate derived from a verbal task. In fact, it appears that the forward digit span obtained may be slightly larger, and the reverse span slightly smaller, when using an up-down (repeated trial) approach in comparison with the traditional (two-trial) verbal method of administration. These "discrepancies" between the two versions of the span estimates may be the cause of the low levels of association evidenced by the correlation coefficients, all falling below even 40 .

An important point should be made concerning the use (and utility) of the Bland-Altman plot (also called a meandifference plot) in contrasting agreement with association. Association is simply a linear relationship, and should be expected if the two measures generally change in the same direction with the same (consistent) rate (i.e., the slope). Conversely, agreement is more stringent, requiring that two measurement methods result in the same (or a very similar) outcome. If two measures of the same underlying construct agree, then the plot of their difference (the average value of which should be zero-so long as they are on the same scale, which they generally are, in the present case) should look like a zero-slope blob along the $x$-axis of their average. Observing this pattern represents an absence of bias in terms of whether or not the measures pro- 
vide different information, depending on the actual level of what is being measured. However, in the Bland-Altman plots based on the online and verbal digit span tasks, the differences do appear to become more negative as the span increases. That is, in Figures 1A and 2A, the online span estimate is less than the span estimate from the verbal test for higher values of the average, resulting in the downward pattern in the Bland-Altman plots. This particular pattern (for forward and reverse spans) is largely absent from the scatterplots (Figures 1B and 2B), which simply reflect the positive association between the two tests of span. How and when the measures do not agree is important information (even for such a small sample as is described here) that is not evident in the scatterplots.

These results are based on very small samples; when replicated with larger samples and possibly across age groups, the online digit span task will allow researchers to differentiate between the capacities of populations to a degree similar to that obtained using the traditional version. The online version of the digit span task provides three desirable features that are not possible in the traditional version: (1) The rate of delivery of the stimuli can be standardized across all subjects; (2) the program can be designed for the collection and comparison of both the stimulus and response strings (e.g., for analyses of primacy and recency effects in verbal immediate memory); and (3) the online version is accessible by any (sighted) subject regardless of hearing status or receptive-language deficit; the instrument can be modified to be accessible to persons with visual deficits as well. However, some level of facility with computers is required, as is the capability to provide a manual response. With the code provided, other researchers can create an online two-trial version to compare it directly with the six-trial version we describe here; the code can also be modified, with the appropriate equipment, to accommodate a verbal response rather than a manual one. These attributes generally translate to the possibility of obtaining more data from more subjects, with a standardized and reliable manner of collection and data entry. We hope that, by making the code freely available (and manipulable with respect to numbers of trials and modality of response or presentation, or both), more extensive neuropsychological tests may be possible that can evaluate not the degree to which the online and verbal tasks agree, but rather the degree to which the online task reflects, or correlates with, other tasks known to depend on memory.

\section{AUTHOR NOTE}

This article could not have been prepared without the assistance of Ted Wright. Thanks are also due the students and staff at the University of California, Irvine, and at Rancho Santiago, Irvine Valley, and Coastline community colleges, in Orange County, California. The data reported in this article represent part of R.E.T.'s doctoral dissertation. This research was supported in part by the U. C. Irvine School of Social Sciences and Department of Cognitive Science. Support was also provided by NIH Grant M01 RR 020359 from the National Center for Research Resources, a Research Starter Grant to R.E.T. from the Pharmaceutical Research and Manufacturer's Association of America Foundation, and an intramural grant to R.E.T. from the Georgetown University Aging Initiative, sponsored by NIA Grant K07 AG019165-03. Correspondence concerning this article should be addressed to R. E. Tractenberg, Department of Neurology, 291 Building D, 4000 Reservoir Road NW, Washington, DC 20057 (e-mail: ret7@georgetown.edu).

\section{REFERENCES}

Altman, D. G., \& Bland, J. M. (1983). Measurement in medicine: The analysis of method comparison studies. Statistician, 32, 307-317.

Bland, J. M., \& Altman, D. G. (1986). Statistical methods for assessing agreement between two methods of clinical measurement. Lancet, $1,307-310$

Gathercole, S. E., \& Baddeley, A. D. (1993). Working memory and language. Hillsdale, NJ: Erlbaum.

LeviTT, H. (1971). Transformed up-down methods in psychoacoustics. Journal of the Acoustical Society of America, 49, 467-477.

Mellard, D. F. (1990). The eligibility process: Identifying students with learning disabilities in California's community colleges. Learning Disabilities Focus, 5, 75-90.

Milner, P. M. (1974). A model for visual shape recognition. Psychological Review, 81, 521-535.

Nunnally, J. C., \& Bernstein, I. H. (1994). Psychometric theory (3rd ed.). New York: McGraw-Hill.

Penney, C. G. (1975). Modality effects in short-term verbal memory. Psychological Bulletin, 82, 68-84.

Stevens, J. (1996). Applied multivariate statistics for the social sciences (3rd ed.). Mahwah, NJ: Erlbaum.

TorgesEn, J. K. (1996). A model of memory from an information processing perspective: The special case of phonological memory. In G. R. Lyon \& N. A. Krasnegor (Eds.), Attention, memory, and executive function (pp. 157-184). Baltimore: Brookes.

Tractenberg, R. E. (1997). Toward an understanding of reading disability: A comparison of the component reading skills of deaf and reading disabled adults. Unpublished doctoral dissertation, University of California, Irvine.

Trojano, L., Chiacchio, L., De Luca, G., Fragassi, N. A., \& Grossi, D. (1994). Effect of testing procedure on Corsi's block-tapping task in normal subjects and Alzheimer-type dementia. Perceptual \& Motor Skills, 78, 859-863.

Tyler, C. W., \& Gorea, A. (1986). Different encoding mechanisms for phase and contrast. Vision Research, 26, 1073-1082.

WeCHSLER, D. (1981). Wechsler Adult Intelligence Scale-Revised. San Antonio, TX: Psychological Corporation.

WechSLER, D. (1983). Wechsler Intelligence Scale for ChildrenRevised. San Antonio, TX: Psychological Corporation.

\section{NOTE}

1. These criteria are not current in identifying reading disability but were in effect when these data were collected.

\section{ARCHIVED MATERIALS}

The following materials associated with this article may be accessed through the Psychonomic Society's Norms, Stimuli, and Data archive, www.psychonomic.org/archive.

To access these files, search the archive for this article using the journal name (Behavior Research Methods), the first author's name (Tractenberg), and the publication year (2007).

FILE: Tractenberg-BRM-2007.zip

DESCRIPTION: The compressed archive file contains one file: Tractenberg.txt, containing digit span test javascript library.

AUTHOR’s E-MAIL ADDRESS: ret7@georgetown.edu. 


\section{APPENDIX}

Task Instructions

\section{Digit Span (Online)}

[single screen with button labeled DONE at the bottom]

Instructions. In this task you will see single numbers that you will have to remember, in order. The first set that you will have to remember will have four numbers between 0 and 9 in it. Each of these numbers will be shown one at a time. At the end of a set of numbers, you will see the cues "FORWARD" or "BACKWARD." As soon as you see the "FORWARD" cue, please type all the numbers, in the exact order that you saw them. Don't use spaces or commas, just the numbers. If you see the "BACKWARD" cue at the end of a set of numbers, you must type all the numbers in the reverse order from how you saw them. For example, if you see 123 [FORWARD], then you would type "1 23 " and hit Return. However, if you see 123 [BACKWARD], then you would write "3 21 ," in that order. It is important that you type the digits in the order indicated.

This task compares your memory for numbers (FORWARD responses) with your memory for the order of numbers (BACKWARD responses).

If you forget one number, put an $\mathrm{x}$ in its place and continue typing your answer without spaces or commas. For the reverse order responses, start with the most recent number and type the set out in reverse order. You will always see either FORWARD or BACKWARD as your cue to type the set you just saw. You will have all FORWARD sequences first, then all BACKWARD sequences. When you click on DONE, the task will begin.

\section{Digit Span (Traditional)}

[single sheet of paper shown to subject to read and listen to as experimenter read]

Instructions. In this task, I will read lists of numbers to you, and then say either "frontward" or "backward." Your job is to repeat the digits back to me in the direction specified. If you need more information, please ask.

Example Responses to Online Digit Span Task: Forward Condition

\begin{tabular}{|c|c|c|c|}
\hline Stimuli & Response & Inflection Counter & Comment \\
\hline 123 & 123 & 0 & Correct answer; no inflections yet. Next trial larger. \\
\hline 1234 & 1234 & 0 & Correct answer; no inflections yet. Next trial larger. \\
\hline 12345 & $12 \times 34$ & 1 & $\begin{array}{l}\text { Incorrect answer; inflection count increased by one } \\
\text { and change of direction in how digit span trial size } \\
\text { is determined. Next trial smaller. }\end{array}$ \\
\hline 1234 & $123 x$ & 1 & $\begin{array}{l}\text { Incorrect answer; no inflection count increase be- } \\
\text { cause performance is in the same (incorrect) direc- } \\
\text { tion. Next trial smaller. }\end{array}$ \\
\hline 123 & 123 & 2 & $\begin{array}{l}\text { Correct answer. Inflection count increases because } \\
\text { performance changed (correct). Next trial larger. }\end{array}$ \\
\hline 1234 & 1234 & 2 & $\begin{array}{l}\text { Correct answer; no inflection count increase be- } \\
\text { cause performance is in the same (correct) direc- } \\
\text { tion. Next trial larger. }\end{array}$ \\
\hline 12345 & $1234 x$ & 3 & $\begin{array}{l}\text { Incorrect answer; inflection count increased by one } \\
\text { and change of direction in how digit span trial size } \\
\text { is determined. Next trial smaller. }\end{array}$ \\
\hline 1234 & 1234 & 4 & $\begin{array}{l}\text { Correct answer; inflection count increased by one } \\
\text { and change of direction in how digit span trial size } \\
\text { is determined. Next trial larger. }\end{array}$ \\
\hline 12345 & $1234 x$ & 5 & $\begin{array}{l}\text { Incorrect answer; inflection count increased by one } \\
\text { and change of direction in how digit span trial size } \\
\text { is determined. Next trial smaller. }\end{array}$ \\
\hline 1234 & 1234 & 6 & $\begin{array}{l}\text { Correct answer; inflection count increased by one } \\
\text { and since inflection count reached six (criterion in } \\
\text { this example), test stops. }\end{array}$ \\
\hline
\end{tabular}

Span estimate for this series is $(5+3+5+4+5+4) / 6=4.33$

(Manuscript received April 19, 2006;

revision accepted for publication February 2, 2007.) 